\title{
Plug-in vs. Wireless Charging: Life Cycle Energy and Greenhouse Gas Emissions for an Electric Bus System
}

\author{
Zicheng Bi ${ }^{\text {a }}$, Lingjun Song ${ }^{\text {b,a }}$, Robert De Kleine ${ }^{a}$, Chunting Chris $\mathrm{Mi}^{\mathrm{c}}$, Gregory A. Keoleian ${ }^{\mathrm{a},{ }^{*}}$ \\ ${ }^{a}$ Center for Sustainable Systems, School of Natural Resources and Environment, University of \\ Michigan, 440 Church St., Ann Arbor, MI 48109, United States \\ b School of Transportation Science and Engineering, Beihang University, Beijing 100191, China \\ ${ }^{\mathrm{c}}$ Department of Electrical and Computer Engineering, University of Michigan-Dearborn, 4901 \\ Evergreen Road, Dearborn, MI 48128, United States
}

\section{Submitted to Applied Energy}

\section{August 25, 2014}

Revision Submitted on February 6, 2015

*Corresponding author. Tel.: +1 7347643194.

Email address: gregak@umich.edu (G.A. Keoleian). 


\begin{abstract}
Wireless charging, as opposed to plug-in charging, is an alternative charging method for electric vehicles (EVs) with rechargeable batteries and can be applicable to EVs with fixed routes, such as transit buses. This study adds to the current research of EV wireless charging by utilizing the Life Cycle Assessment (LCA) to provide a comprehensive framework for comparing the life cycle energy demand and greenhouse gas emissions associated with a stationary wireless charging all-electric bus system to a plug-in charging all-electric bus system. Life cycle inventory analysis of both plug-in and wireless charging hardware was conducted, and battery downsizing, vehicle lightweighting and use-phase energy consumption were modeled. A bus system in Ann Arbor and Ypsilanti area in Michigan is used as the basis for bus system modeling. Results show that the wirelessly charged battery can be downsized to $27-44 \%$ of a plug-in charged battery. The associated reduction of $12-16 \%$ in bus weight for the wireless buses can induce a reduction of 5.4-7.0\% in battery-to-wheel energy consumption. In the base case, the wireless charging system consumes $0.3 \%$ less energy and emits $0.5 \%$ less greenhouse gases than the plug-in charging system in the total life cycle. To further improve the energy and environmental performance of a wireless charging electric bus system, it is important to focus on key parameters including carbon intensity of the electric grid and wireless charging efficiency. If the wireless charging efficiency is improved to the same level as the assumed plug-in charging efficiency (90\%), the difference of life cycle greenhouse gas emissions between the two systems can increase to $6.3 \%$.
\end{abstract}

Keywords: Wireless charging; Plug-in charging; Life cycle assessment; Vehicle lightweighting; Energy; Greenhouse gases 


\section{Introduction}

The transportation sector is responsible for $27 \%$ of U.S. greenhouse gas (GHG) emissions [1] and 28\% of total U.S. energy use [2]. Vehicle electrification through electric vehicles (EVs) with rechargeable batteries has the potential to significantly reduce the GHG emissions compared to a fleet of internal combustion engine vehicles (ICEVs) [3]. Conventional EVs are charged through plug-in chargers, but these EVs face challenges including 1) heavy battery packs, 2) high battery costs, and 3) the inconvenience and time requirements for charging. Heavy battery pack is a critical challenge for further improving vehicle fuel economy, especially for all-electric buses that have large batteries. The battery pack can comprise about $26 \%$ of the weight of bus, considering the example of a long-range all-electric bus manufactured by BYD Auto Company which has a $324 \mathrm{kWh}$ lithium iron phosphate (LFP) battery (assuming 88 $\mathrm{Wh} / \mathrm{kg}$ battery pack) and curb weight of $14 \mathrm{t}[4,5]$. Due to the large size and the high price of lithium material, the LFP battery cost can be as high as $39 \%$ of the total cost of a long-range all-electric bus $[4,6]$.

An alternative charging method, the EV wireless charging, an application of the Wireless Power Transfer (WPT) technology, may overcome the problems of plug-in charging. The WPT technology can be traced back to a century ago when Nicola Tesla introduced near-field coupling of two loop resonators based on magnetic resonance $[7,8]$. With WPT technology, the EV can be charged without a cable and connector. Through the magnetic field between two coil plates, one loaded on the bottom of the vehicle and the other embedded in pavement, the electric energy can be transferred wirelessly. Wireless charging can be classified as stationary or dynamic charging [9]. Stationary wireless charging equipment can be utilized in a garage, parking lot or bus stop. For dynamic charging, the vehicle can be charged in motion through multiple sets of coils and accessories embedded along the road. The charging efficiency of more than $80 \%$ has been reported for both stationary and dynamic charging [9-13]. Currently, wireless charging has been mostly demonstrated on vehicles with fixed routes, such as public transit buses [14].

Life Cycle Assessment (LCA) is the methodology to evaluate the potential environmental impacts associated with the total life cycle of a product or system, which encompasses material production, manufacturing, use and retirement stages [15]. LCA can help researchers better understand the wireless charging EV system from energy and environmental perspectives. Life cycle energy demand and GHG emissions are two metrics for evaluation in this study.

Wireless charging provides frequent charging opportunities at transit centers and major bus stops during bus operation hours. This can lead to battery downsizing, which results in vehicle lightweighting and fuel economy improvement, compared with plug-in 
charging. Associated benefits may include reduced energy consumption and emissions in battery production and potential reduction in use-phase electricity consumption for a pure electric vehicle. However, the wireless charging infrastructure can create additional energy and environmental burdens. Thus, it is meaningful to analyze the tradeoffs and inform future development of wireless charging bus systems.

This paper compares plug-in and stationary wireless charging from a life cycle perspective, based on an existing transit bus system to evaluate the energy consumption and GHG emissions. Although there is significant ongoing research into the engineering side of EV wireless charging [10, 13, 16-19], research examining life cycle energy and environmental implications is not well established. This study adds to the current development of EV wireless charging by utilizing LCA methods to model the plug-in and wireless chargers, battery downsizing and use-phase lightweighting benefits. In addition, this study highlights key parameters that greatly influence the energy and GHG emissions of a wireless charging bus system.

\section{Method}

\subsection{Goal and Scope}

The goal of this LCA study is to compare two charging scenarios for an all-electric bus system, plug-in charging and stationary wireless charging, in terms of Cumulative Energy Demand (CED, TJ) [20, 21] and 100-year Global Warming Impact (GWI, t $\mathrm{CO}_{2-}$ eq) [22]. CED represents total primary energy requirements of both renewable and nonrenewable sources, including fossil, nuclear, biomass, wind, solar, geothermal energy and hydropower. This study models the major differences between the two systems using a process-based LCA approach in order to quantify the burdens associated with each stage of the life cycle, including the material extraction, production and manufacturing burden of chargers and batteries, as well as the use-phase energy consumption. The end-of-life stage is excluded due to lack of data. It is assumed that the buses in each charging scenario are all-electric and made with identical components, except for battery and charger. So the materials and manufacturing of the bus shell and other accessories are not modeled. Stationary wireless charging is considered as the wireless charging method in our model.

An existing transit bus system serving the Ann Arbor and Ypsilanti area in Michigan, USA, called TheRide [23], is used as the basis for our bus system simulation. The total numbers of routes, buses and bus stops for the model are adapted from the current bus system. Only regular transit routes are considered. Altogether sixty-seven buses and twenty-one routes are modeled. The adapted bus system map and the modeling parameters can be found in the Supporting Information (Figure S1, Table S1 and Table S2). The twenty-one routes are classified into three groups for simplicity: the blue (Ann 
Arbor city routes), red (Ann Arbor-Ypsilanti intercity routes) and green routes (Ypsilanti city routes), based on their service areas. Thirteen blue routes operate in Ann Arbor downtown and its suburban area, four red routes operate between Ann Arbor and Ypsilanti downtowns and their suburban areas, and four green routes operate in Ypsilanti downtown and its suburban area. Routes in the same group are assumed to have the same parameters, such as travel distance in each loop. It is assumed that about one quarter of bus operation time (from the beginning to end of daily duty) is dwell time at bus stops and transit centers, which is based on a study for a typical route in the TheRide bus system [24].

Two charging scenarios of this bus system are modeled: 1) plug-in charging scenario and 2) stationary wireless charging scenario. For the plug-in charging scenario, the plugin chargers are assumed to be only located at the parking lot for buses to charge overnight. For the wireless charging scenario, the wireless charging infrastructure is hypothesized to be deployed across the bus service area located at those popular bus stops where the buses stop more frequently, all of the transit centers, as well as the overnight parking lot. It is assumed that the downtown areas have greater charging infrastructure density than the suburban areas. A longer charge time is assumed at each transit center (e.g. 6 minutes) and key bus stop (e.g. 36 seconds per charging stop) in downtown than the suburban areas (e.g. 29 seconds per charging stop) because the ridership is assumed to be lower in suburb. The result is that about one quarter of the operation time is dwell time for wireless charging.

The functional unit for this study is providing transit services for Ann Arbor and Ypsilanti area for 12 years with 67 buses, equivalent to 48,034,407 vehicle kilometers in total (on average 716,932 vehicle kilometers per bus). The 12-year horizon is often used as an estimate for bus life in transit agency reports, such as U.S. Federal Transit Administration [25], and used by a previous LCA study modeling electric public transportation buses [26].

\subsection{Data Sources and Model Description}

\subsubsection{Inventories of Plug-in and Wireless Chargers}

Figure 1 depicts and compares the wireless charger (WC) and plug-in charger (PC) components. The charger components are modeled based on a $6 \mathrm{~kW}$ wireless charger currently under development at the University of Michigan-Dearborn [10]. The plug and cable of a plug-in charger are modeled based on a 2013 Chevrolet Volt charger. The mass

of charger components was measured and then modeled using the LCA software SimaPro 8 [27]. To transmit $60 \mathrm{~kW}$ of power to charge the bus, it is assumed that 10 sets of $6 \mathrm{~kW}$ chargers are installed and the material burden is scaled up proportionally. A $60 \mathrm{~kW}$ wireless charger is separated into on-board portion (on-WC) and off-board portion, i.e., 
those installed on the ground (off-WC). In total, there are 67 buses operating on 21 routes, with 67 batteries and 67 on-WCs installed on buses. A total of 428 off-WCs ( $60 \mathrm{~kW}$ each) located at major bus stops, transit centers and the parking lot are modeled. Both wireless and plug-in chargers are assumed to last 24 years, thus half of the burdens are allocated to the future and not considered in our 12-year horizon. Details on charger life estimation are given in the discussion section and charger inventories are available in the Supporting Information (Section 2).

(a) Wireless Charger

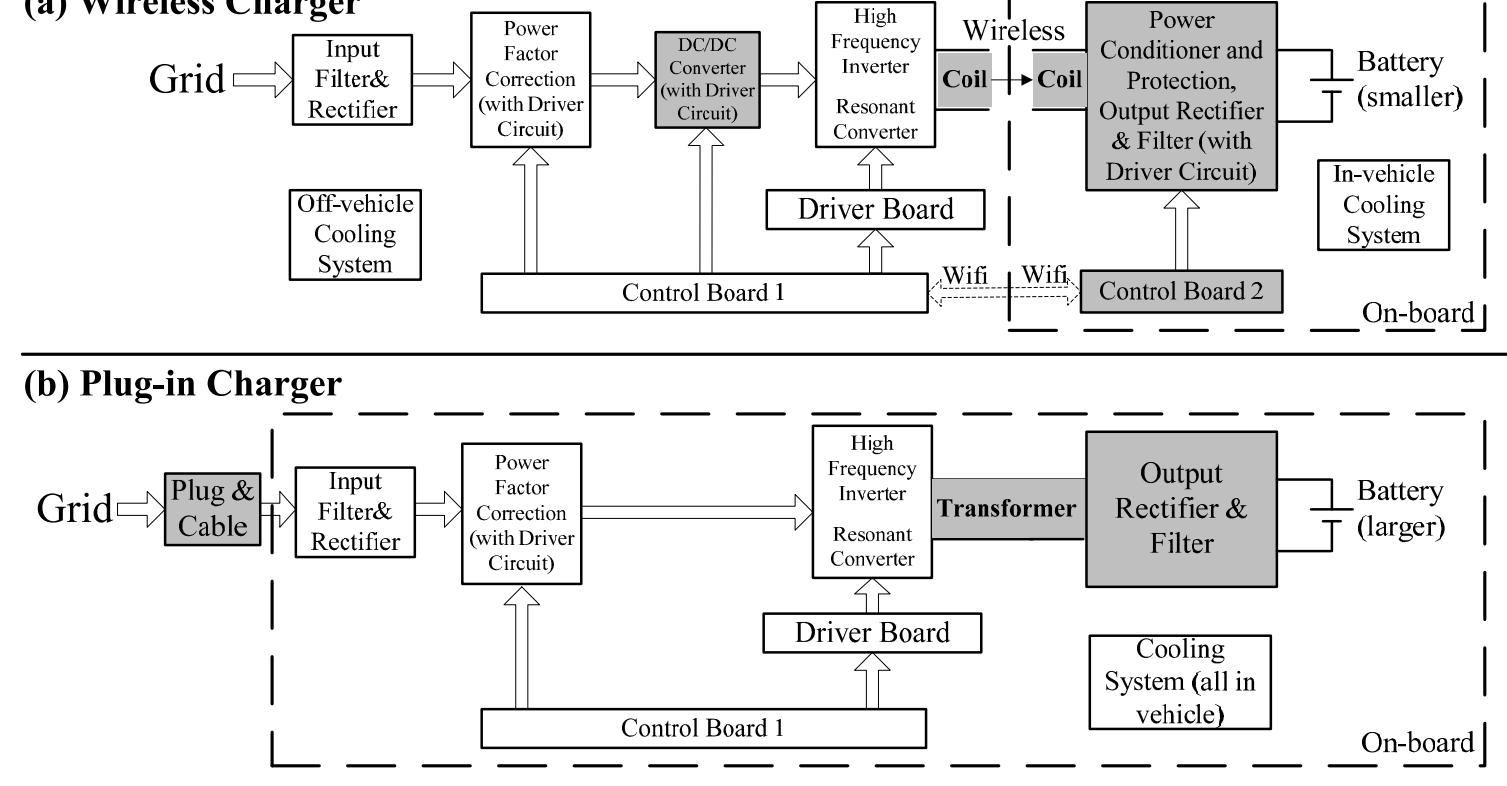

Figure 1. Comparison of wireless charger (a) and plug-in charger (b) for electric vehicles. Component difference is marked in grey. On-board portion of each charger is in the dashed box, and the rest is outside the vehicle. This graph is based on a $6 \mathrm{~kW}$ wireless charger from Professor Chris Mi's laboratory in University of Michigan, Dearborn.

\subsubsection{Battery Downsizing and Lightweighting Calculation}

The wireless battery can be downsized due to multiple charges during daily operation, which leads to a reduction of bus weight. The battery downsizing and lightweighting calculation for the wireless charging scenario relative to the plug-in charging scenario is illustrated in Figure 2. The capacity of a plug-in charged battery can be divided into four regions: Overcharge Safety Margin (OSM), Operating Region (OR), Reserved Storage Capacity (RSC) and No Operation Region (NOR) [28]. The electricity amount represented by $O R(\mathrm{kWh})$ indicates the minimum battery energy requirement at start of each day for a bus to fulfill its daily duty. The State of Charge Range $(S O C R, \%)$ is defined as the percentage of the $O R(\mathrm{kWh})$ relative to the whole capacity of a new battery $(\mathrm{kWh})$. The RSC serves two functions: as an energy reserve for unexpected energy demand during daily operation, as well as battery capacity fade in the future. The 
electricity in a plug-in charged battery depletes at an energy consumption rate (ECR) of $k_{1}(\mathrm{kWh} / \mathrm{km})$.

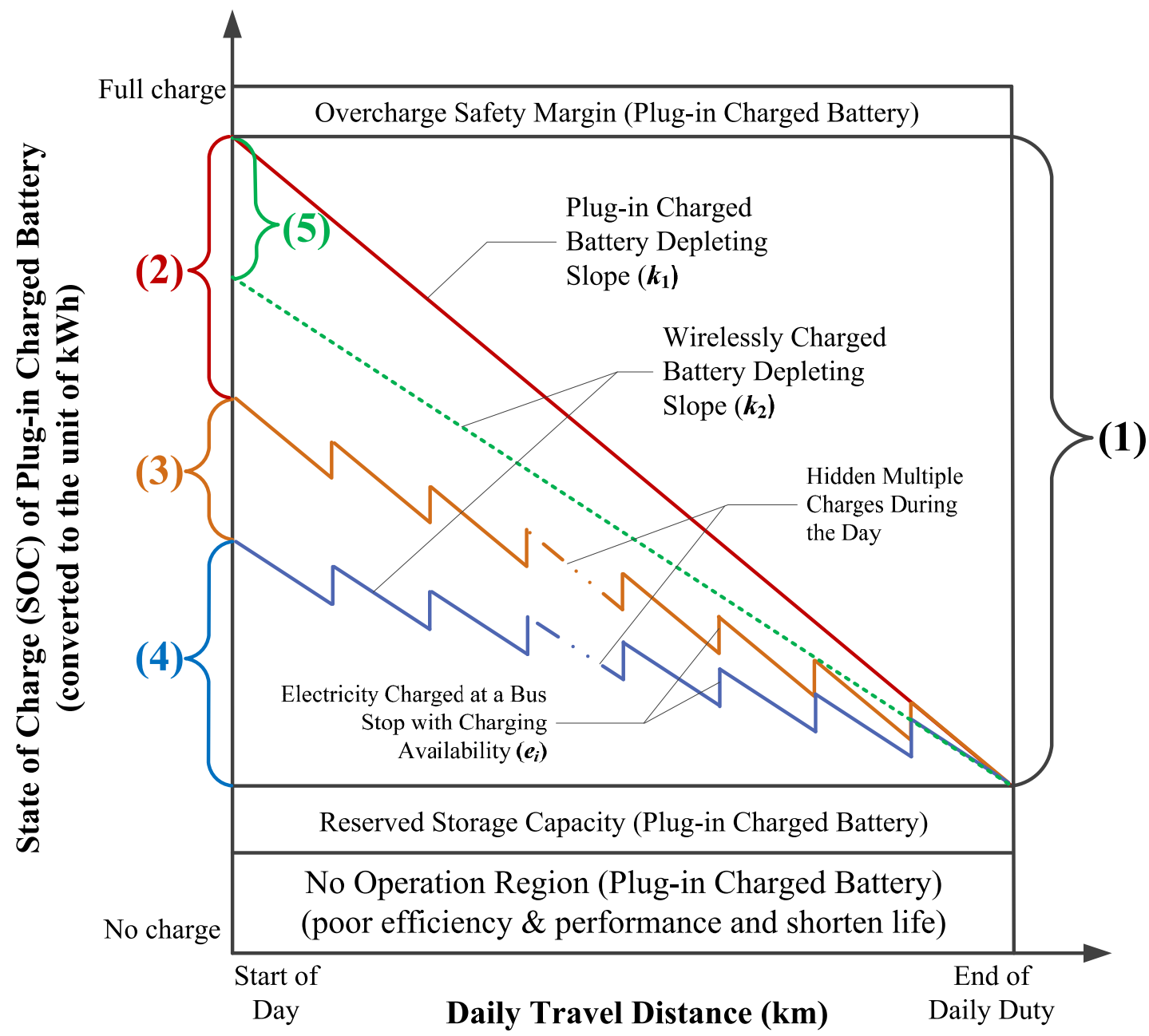

Figure 2. Battery downsizing calculation. Start from a plug-in charged battery to quantify the minimum electricity needed at start of day for a wirelessly charged battery. (1) Operating Region (kWh) of plug-in charged battery; (2) Primary capacity reduction (kWh) due to wireless charging availability; (3) Secondary capacity reduction (kWh) due to fuel economy improvement; (4) Minimum electricity needed (kWh) at start of day for a wirelessly charged battery (not yet including its own Overcharge Safety Margin, Reserved Storage Capacity and No Operation Region); (5) Daily energy saving (batteryto-wheel) due to wireless charging (kWh).

The plug-in charged battery can first be downsized due to wireless charging availability at charging stations. Since the bus can charge at each of those stations, it is 
reasonable for the bus to carry a smaller battery to travel the same distance than the plugin charging scenario, which results in the primary capacity reduction. The battery capacity after primary capacity reduction $\left(C_{1}, \mathrm{kWh}\right)$ can be calculated from Eq. (1) and Eq. (2), where $E_{1}(\mathrm{kWh})$ is the total amount of electricity charged during operation hours, $e_{i}(\mathrm{kWh})$ is the amount of electricity charged at charging stop $i, N$ is the total number of stops where the bus stops for charging during the operation, $\eta(\%)$ is the average charging efficiency, $P(\mathrm{~kW})$ is the charging power and $T$ (hour) is the total amount of charging time at charging stops during the day. The SOCR for the wirelessly charged battery is the same as that of the plug-in charged battery $(60 \%)$. The battery weight $(\mathrm{kg})$ can be calculated by dividing the battery capacity $(\mathrm{kWh})$ by the battery specific energy $(0.13$ $\mathrm{kWh}$ per kg of Li-ion battery) [29]. Therefore, the percentage of vehicle mass reduction due to primary downsizing can be determined, relative to a bus of $15 \mathrm{t}$, comprising the assumed curb weight of $14 \mathrm{t}$ [4] and the constant average weight of $1 \mathrm{t}$ for driver, passengers and cargo [30]. Fluctuation of ridership is not considered.

$$
\begin{aligned}
& E_{1}=\sum_{i=1}^{N} e_{i}=\eta P T \\
& C_{1}=\frac{O R-E_{1}}{S O C R}
\end{aligned}
$$

Vehicle lightweighting due to the primary battery downsizing will improve the fuel economy, which means the electricity in a wirelessly charged battery will deplete more slowly than a plug-in charged battery $\left(\left|k_{2}\right|<\left|k_{1}\right|\right)$. The ECR of a wirelessly charged bus, $k_{2}$ $(\mathrm{kWh} / \mathrm{km})$, can be determined by the lightweighting correlation. For a sedan, $10 \%$ vehicle mass reduction results in a 6-8\% use-phase energy reduction for an Internal Combustion Engine Vehicle (ICEV) and 4-7\% for many EV types and models [31, 32]. The EVs are less sensitive to the mass-induced energy consumption reduction due to the regenerative braking and higher powertrain efficiency [33], so the percentage change in energy consumption of EV is slightly lower than that of ICEV. For a bus, $10 \%$ vehicle mass reduction results in about 5\% energy reduction for a conventional bus in the Autonomie model [34]. However, the lightweighting correlation of an all-electric bus is not available. The percentage change in energy consumption of an electric bus is assumed to be $10 \%$ lower than that of a conventional bus, thus $4.5 \%$ energy reduction per $10 \%$ vehicle mass reduction for a pure electric bus is assumed for the baseline case. This assumption is further analyzed in the sensitivity analysis section. A lighter bus will consume less energy to cover its daily travel distance, which results in the secondary downsizing of the battery. The energy saving $\left(E_{2}, \mathrm{kWh}\right)$ related to secondary downsizing can be calculated from Eq. (3), where DTD $(\mathrm{km})$ is the daily travel distance.

$$
E_{2}=\left|k_{1}-k_{2}\right| D T D
$$


Deducting primary $\left(E_{1}, \mathrm{kWh}\right)$ and secondary $\left(E_{2}, \mathrm{kWh}\right)$ capacity reductions from the $O R(\mathrm{kWh})$ of the plug-in charged battery, the minimum electricity needed at the start of day for a wirelessly charged battery can be quantified. Dividing this value by the SOCR $(60 \%)$, the capacity of a wirelessly charged battery $\left(C_{2}, \mathrm{kWh}\right)$, including the OSM, RSC and NOR, can be quantified. Dividing the battery capacity $C_{2}(\mathrm{kWh})$ by the battery specific energy $(\mathrm{kWh} / \mathrm{kg})$, the final weight $(\mathrm{kg})$ of the wirelessly charged battery can be determined. The fuel economy can be further improved, which will further downsize the battery. Further iterations of fuel economy and battery downsizing are smaller compared to primary and secondary effects, and so are ignored in this model, which results in a conservative estimate for the wirelessly charged battery.

The lithium-ion battery with $\mathrm{LiMn}_{2} \mathrm{O}_{4}(\mathrm{LMO})$ cathode material is used to model both plug-in and wirelessly charged batteries. LMO was chosen because of its well established life cycle inventory, lower cost and abundance of manganese in nature [35, 36]. The cradle-to-gate energy and GHG emissions are modeled as $75 \mathrm{MJ} / \mathrm{kg}$ battery and $5.1 \mathrm{~kg}$ $\mathrm{CO}_{2}$-eq/kg battery, according to a process LCA study of LMO batteries for electric vehicles that is specific to the United States [29]. When the OSM is 5\%, RSC is $15 \%$ and NOR is $20 \%$, the SOCR is $60 \%$ with the state of charge (SOC, \%) assumed to swing around $35 \%$ to $95 \%$ for both plug-in and wirelessly charged batteries [28]. The cycle life of battery is assumed to be 3000 cycles, based on the general performance of lithium-ion batteries $[5,26,37]$. For a wirelessly charged battery, the multiple wireless charges during daily operation, however, are assumed to have negligible effect on the battery life. Therefore, for both plug-in and wirelessly charged batteries, energy depleting from $95 \%$ to $35 \%$ SOC during the operation and charging back to $95 \%$ overnight are assumed to represent one cycle.

\subsubsection{Use-phase Energy Consumption}

The life cycle energy and GHG emission intensities of electricity are from U.S. Life Cycle Inventory's (USLCI) low-voltage grid average data for the United States. The Cumulative Energy Demand (CED) is $10.9 \mathrm{MJ} / \mathrm{kWh}$ of electricity delivered and the 100year Global Warming Impact (GWI) is $0.784 \mathrm{~kg} \mathrm{CO}_{2}$-eq/kWh of electricity delivered [38]. In the base case, the same CED and GWI intensities for the electric grid are used for both nighttime and daytime charging. The variation in electricity carbon intensity between day and night is further explored in a sensitivity analysis.

The life cycle energy demand is calculated based on a series of energy transmission and conversion efficiencies illustrated in Figure 3. Energy is converted and transmitted from resource energy to wheels, with energy losses at each step. At the first step, from resource energy to bus stop, there assumes to be an efficiency of 33\%, including extraction, transport, generation and delivery through electric grid, calculated from 10.9 $\mathrm{MJ} / \mathrm{kWh}$ mentioned in the previous paragraph [38]. The plug-in charger efficiency for an 
electric bus is assumed to be $90 \%$ [26]. The wireless charging efficiency is assumed to be $85 \%$ [9-12]. The wireless charging efficiency includes the energy losses due to potential misalignment of on-board and off-board charging pads and the charger design itself. The lithium-ion battery charge/discharge efficiency is assumed to be $90 \%[5,39]$. The battery-to-wheel energy consumption rate of the plug-in charged bus $\left(k_{1}\right)$ is assumed to be $1.46 \mathrm{kWh} / \mathrm{km}(=2.35 \mathrm{kWh} / \mathrm{mile})$, estimated from several trial and demo operations of the BYD all-electric bus [4]. The ECR of the wirelessly charged bus $\left(k_{2}, \mathrm{kWh} / \mathrm{km}\right)$ can be quantified by the battery downsizing calculation. Finally, with the lifetime kilometers traveled, the life cycle energy demand can be quantified.

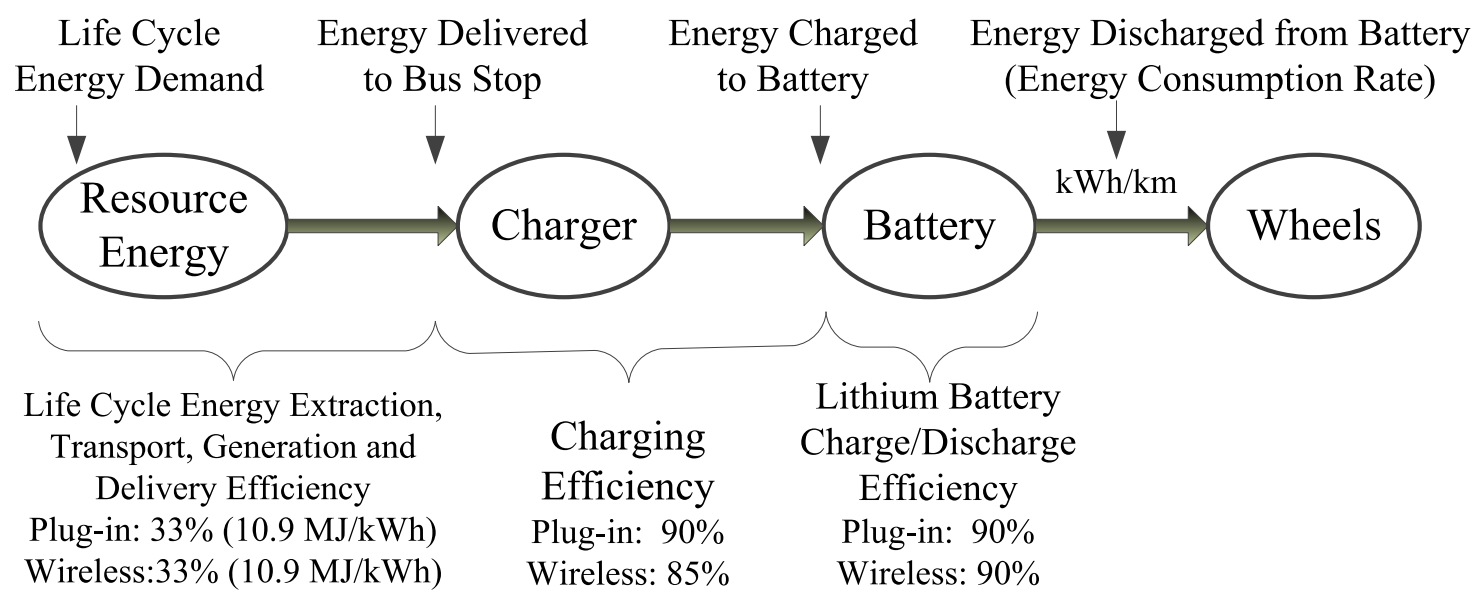

Figure 3. Life cycle energy transmission and conversion cascade.

\section{Results}

\subsection{Cumulative Energy Demand and Global Warming Impact}

The CED and GWI results for the base case are shown in Figure 4 (a) and (b), respectively. The graphs show the life cycle energy demand and GHG emissions of the whole bus system across the service area, either with plug-in charging or wireless charging, within a 12-year period. There is not much difference found comparing the plug-in and wireless charging systems from the perspectives of CED and GWI. Wireless charging system consumes $0.3 \%$ less energy and emits $0.5 \%$ less GHGs than plug-in charging system in the total life cycle. The tradeoffs of increased energy and GHG burdens from wireless charging infrastructure and benefits from battery downsizing are clearly illustrated. 


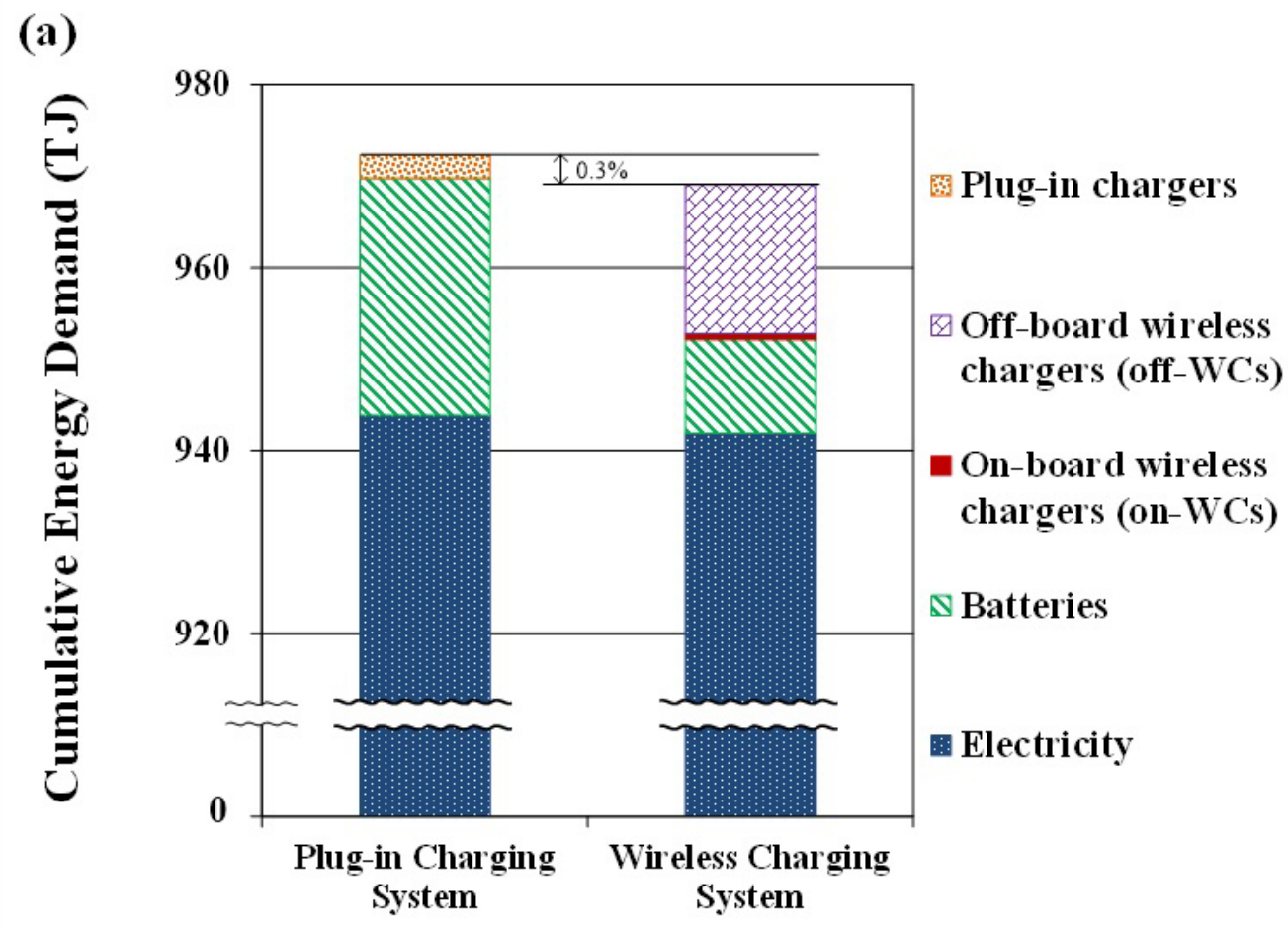

(b)

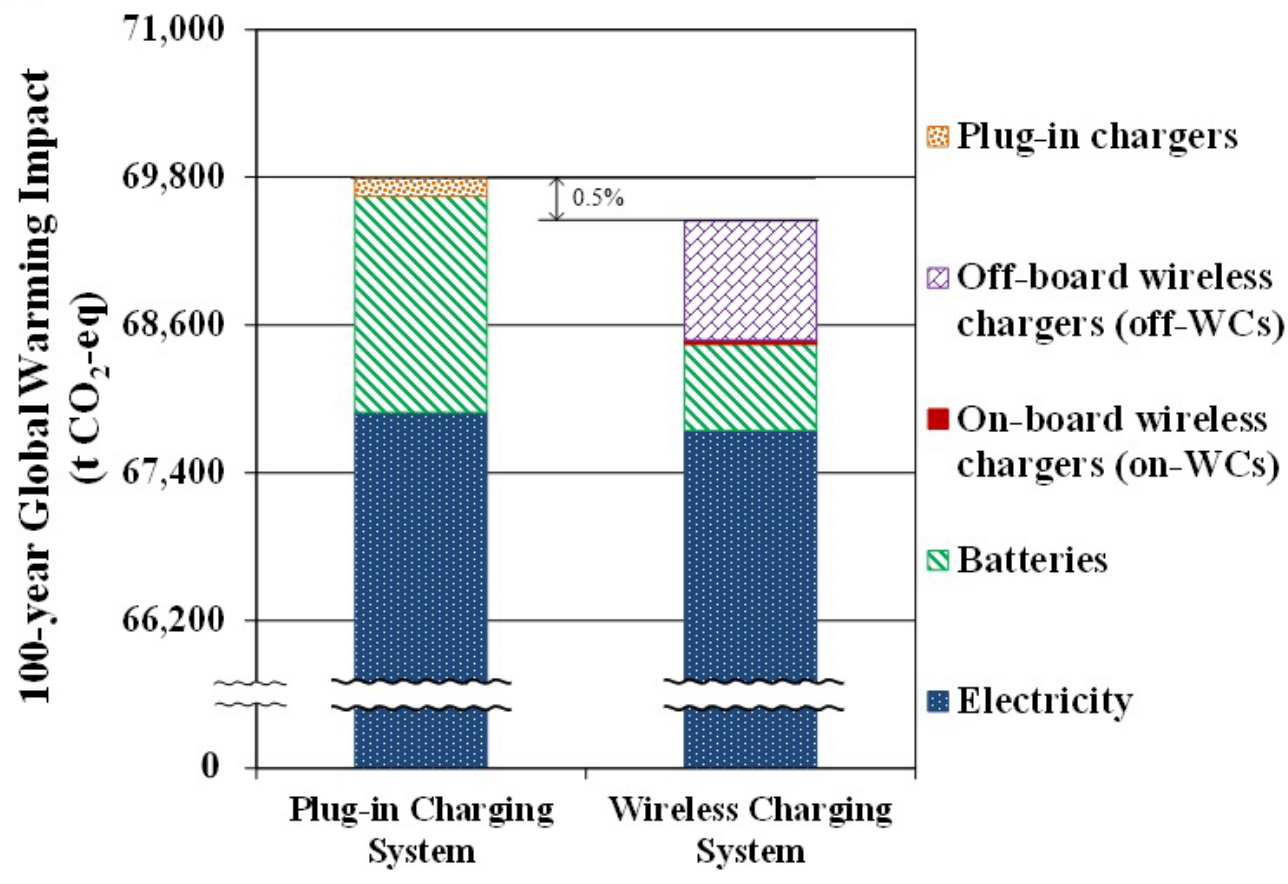

Figure 4. Cumulative Energy Demand (a) and 100-year Global Warming Impact (b) of plug-in and wireless charging electric bus systems.

For charger production, one $60 \mathrm{~kW}$ plug-in charger consumes $0.075 \mathrm{TJ}$ life cycle energy and emits life cycle GHGs of $4.56 \mathrm{t} \mathrm{CO}_{2}$-eq. One $60 \mathrm{~kW}$ on-WC consumes 0.018 
$\mathrm{TJ}$ and emits $1.03 \mathrm{t} \mathrm{CO}_{2}$-eq and one $60 \mathrm{~kW}$ off-WC consumes $0.076 \mathrm{TJ}$ and emits $4.53 \mathrm{t}$ $\mathrm{CO}_{2}$-eq. Based on the assumptions of the bus system, there are 428 off-WCs and 67 onWCs deployed for the wireless charging system, while the plug-in charging system only requires 67 plug-in chargers for charging each bus overnight. As a result, the energy and GHG emissions from all chargers (on-WCs and off-WCs) in the wireless charging scenario are respectively 6.7 and 6.6 times greater than from all plug-in chargers in the plug-in charging scenario.

The weights of both plug-in and wireless batteries as well as the corresponding ECRs are shown in Figure 5. It is assumed that each bus travels the same distance each weekday. Based on the daily energy requirement (kWh) and SOCR (\%), the battery weight of a plug-in charged bus is quantified as 3,525 kg (458 kWh). For a wireless bus, the more charging is available during the day, the more the battery could be downsized. Results show that the buses in the red routes (Ann Arbor-Ypsilanti intercity routes) have potential to carry smaller batteries and have lower ECR, because they have longer charging time during operation than the blue and green routes. The battery can be downsized to $27 \%$ of the plug-in charged battery for the red routes, and $44 \%$ for the blue and green routes. Based on the cycle life of the battery, the batteries are replaced about every 8 years for both types of buses, so the energy and environmental burden of one and a half batteries is counted in the 12-year lifetime. As shown in Figure 4 (a) and (b), after aggregating all the batteries in the whole bus system and taking into account the battery replacement, the energy demand and GHG emissions related to battery material production and manufacturing in the wireless charging system are about $40 \%$ of those in a plug-in charging system. 


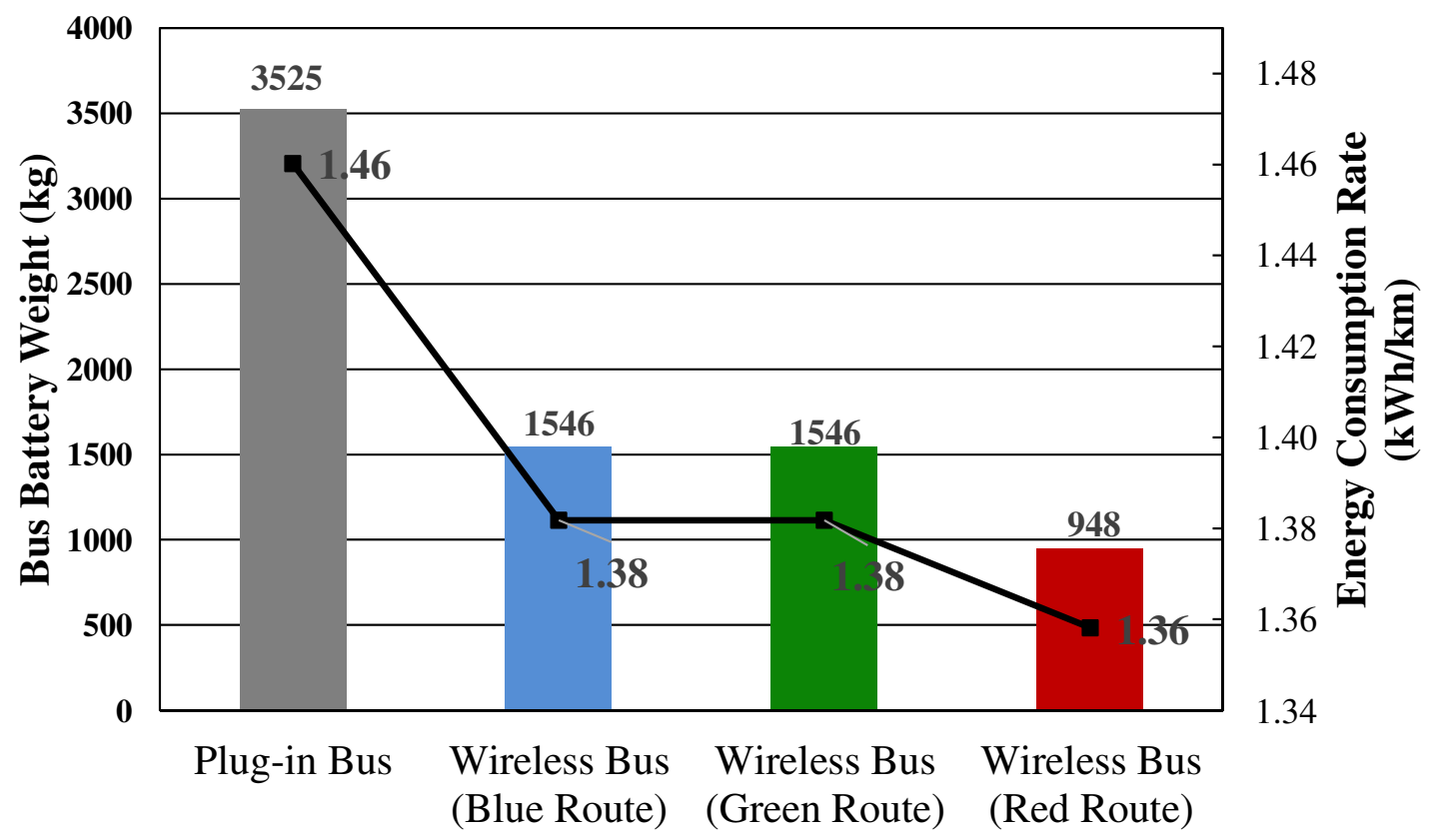

Figure 5. Battery weights and energy consumption rates for plug-in and wirelessly charged buses (the columns are in primary y-axis on the left and the line is in secondary $\mathrm{y}$-axis on the right). Blue routes = Ann Arbor city routes; red routes = Ann ArborYpsilanti intercity routes; green routes $=$ Ypsilanti city routes.

The use-phase electricity consumption dominates the life cycle energy demand and GHG emissions, accounting for about 97-98\% of CED and GWI. The lightweighting benefit of wireless charging can be offset by its current relatively poorer charging efficiency, so there is not much difference in electricity consumption between the plug-in and wireless charging systems from the perspectives of CED and GWI. A reduction of $12 \%$ to $16 \%$ in bus weight for the wireless buses induces a reduction of $5.4 \%$ to $7 \%$ in batteryto-wheel energy consumption rate. However, the wireless charging efficiency is assumed to be $85 \%$, compared to $90 \%$ for a plug-in charger. Therefore, though the battery-towheel ECR $(\mathrm{kWh} / \mathrm{km})$ is smaller for a lightweight bus, the electricity charged to the bus will not necessarily be smaller and is highly dependent on the charging efficiency.

\subsection{Sensitivity Analysis of Key Parameters}

Results of sensitivity analysis are summarized in Figure 6. Of all the parameters tested, the results are most sensitive to the wireless charging efficiency and least sensitive to the wireless battery charge/discharge cycle per day. 


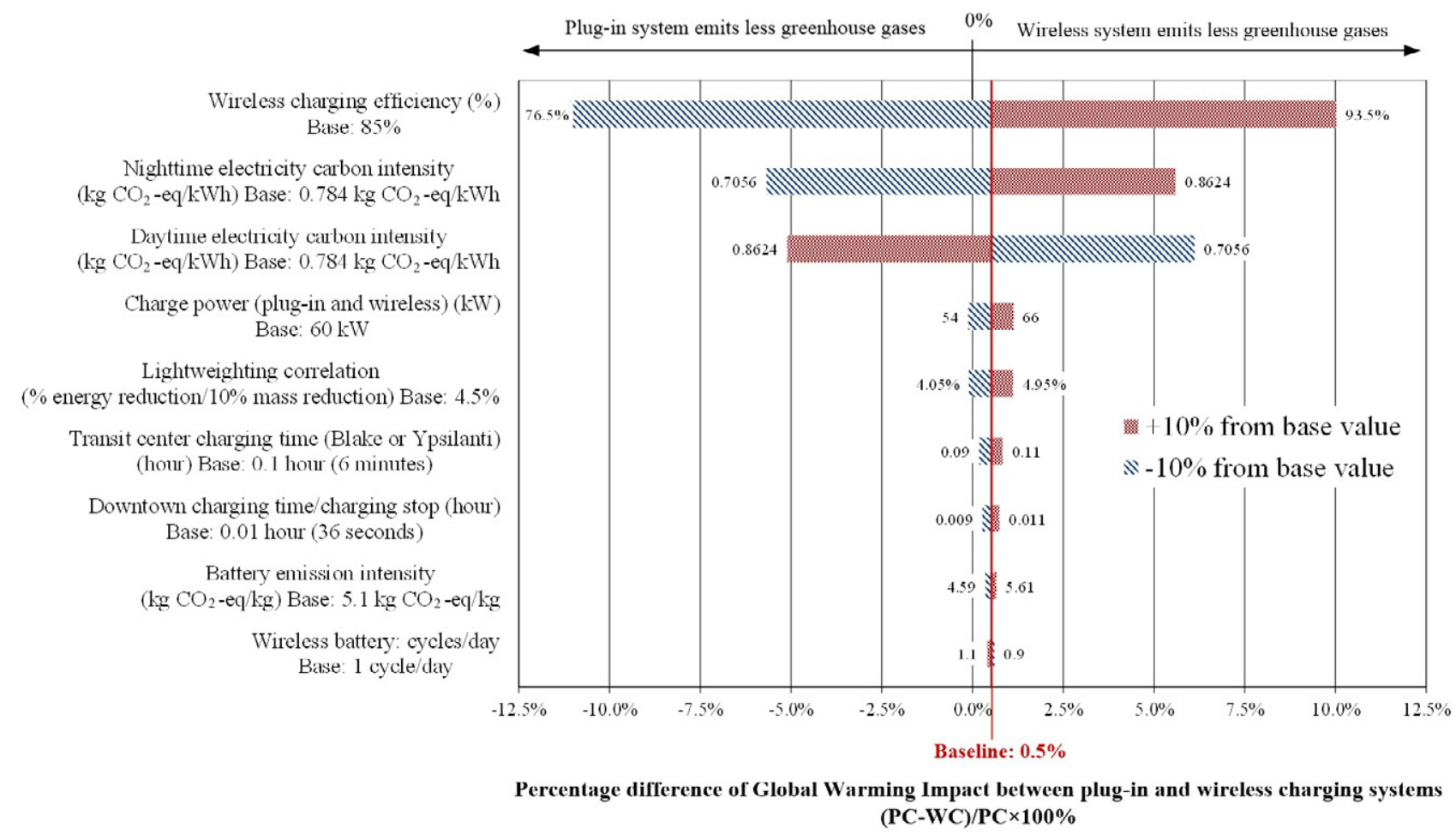

Figure 6. Sensitivity analysis of key parameters. Parameters are ranked according to sensitivity. $(\mathrm{PC}=$ greenhouse gas emissions from plug-in charging system; $\mathrm{WC}=$ greenhouse gas emissions from wireless charging system)

Improving wireless charging efficiency is a very effective way to improve the environmental performance of the wireless charging system from a GWI perspective. If it is improved to the same level as the assumed plug-in charging efficiency (90\%), the life cycle GHG emissions difference between the two systems will be increased to $6.3 \%$.

Results are also sensitive to the carbon intensities of electric generation during the day and night. Based on the model assumptions, the plug-in bus is only charged overnight, but for a wireless bus, about $58 \%$ of the use-phase electricity consumption is charged during operation and about $42 \%$ is charged overnight. The electricity carbon intensity is assumed to be $0.784 \mathrm{~kg} \mathrm{CO}_{2}$-eq/kWh regardless of day and night in the base case. However, due to the different electricity demand between day and night, the fuel profile used for generation changes, resulting in different grid carbon intensities between day and night. Thus, changes in grid carbon intensity are explored separately for day and night in the sensitivity analysis. If daytime electricity carbon intensity decreases by $10 \%$, the wireless charging system emits $6.1 \%$ fewer GHGs than the plug-in charging system. Although the difference in life cycle GHG emissions between the two charging systems is sensitive to the difference of daytime and nighttime grid carbon intensities, it is 
insensitive to simultaneous increase or decrease in grid carbon intensity for both day and night. For example, in some states in the U.S. or some European countries, the grid carbon intensity may be as low as 300-400 $\mathrm{g} \mathrm{CO}_{2}-\mathrm{eq} / \mathrm{kWh}$ or even lower due to more renewable energy integration [26, 40]. If $0.3 \mathrm{~kg} \mathrm{CO}-\mathrm{eq} / \mathrm{kWh}$ is used as both day and night grid carbon intensity instead of the U.S. average of $0.784 \mathrm{~kg} \mathrm{CO}$-eq/ $/ \mathrm{kWh}$, the difference in life cycle carbon emissions between the plug-in charging and wireless charging systems only increases from $0.5 \%$ to $0.9 \%$. Therefore, it is the relative value of the difference in day and night grid carbon intensities rather than the absolute value of the overall level of grid carbon intensity that differentiates the life cycle GHG emissions of the two charging systems.

Parameters including charge power, charging time, life cycle carbon intensity associated with battery production and lightweighting correlation also affect the life cycle GHG emissions. However, the results are less sensitive to these parameters compared with the wireless charging efficiency and electricity carbon intensity.

The wireless battery is charged and discharged more frequently than the plug-in battery, while SOCR is kept constant for both batteries (60\%). With the same SOC window, frequent charge and discharge may have negative impact on battery life. However, the extent of the impact is not yet found in literature. The wireless battery may degrade more quickly and battery replacement may be more frequent. To analyze this impact, it is assumed that a plug-in battery has one charge and discharge cycle per day, while wireless battery may have an equivalent of more than one cycle per day, such as 1.1 cycles per day. However, the results are less sensitive to this parameter than other parameters.

The sensitivity analysis evaluates key input parameters by changing their values individually. If these parameters were changed simultaneously, the wireless charging system could emit considerably less GHG emissions than the plug-in charging system. For example, the wireless charging system would emit 18.6\% less GHGs than the plug-in charging system when the following conditions are met simultaneously: 1) a wireless charging efficiency of $93.5 \%$; 2) the daytime carbon intensity is $10 \%$ lower than nighttime; 3) a charging rate of $66 \mathrm{~kW}$; 3) a lightweighting correlation of $4.95 \%$ fuel reduction per $10 \%$ vehicle mass reduction, 4) a $10 \%$ longer dwell time at charging stations in downtown and transit centers.

\section{Discussion and Conclusion}

Implementing wireless charging for electric buses or other EVs may pose challenges for the electric grid. On one hand, due to the convenience of wireless charging, EVs can be charged during the daytime when the grid faces peak load demand, which requires extra grid supply. Thus, wireless charging during daytime may not help ease the peak 
load power burden of the grid. On the other hand, the life cycle GHG difference between the two systems depends on the fuel mix dispatch of generating assets. If daytime electricity carbon intensity decreases, the wireless charging system emits fewer GHGs than the plug-in charging system. Thus, considering the grid mix difference between day and night, there may be the potential for further reductions in life cycle GHG emissions for the wireless charging EV system.

The battery can be further downsized if more wireless charging infrastructure is implemented. Stationary wireless charging is modeled in this study, which shows a potential of downsizing the battery to about one-third the weight of a plug-in charged battery. If one percent of the buses' routes is covered by off-board wireless charging infrastructure, the battery can be reduced to less than one-fifth the size of the one in a conventional pure electric bus, according to a real world test in Gumi City in Korea [41]. Additionally, the battery SOC swings from 40-60\% for dynamic charging (i.e. charging while in motion) [14], instead of $35-95 \%$ as modeled in this study for stationary charging. Although the battery can be further downsized, the battery cannot be too small in order to maintain a low C-rate for battery health. C-rate is the rate at which a battery is discharged relative to its maximum capacity. For example, $1 \mathrm{C}$ rate means one full battery will be discharged in one hour. If the battery is too small, the C-rate will be high and the battery will degrade faster. Maintaining a low $\mathrm{C}$-rate, such as less than $1 \mathrm{C}$, ensures the battery health and service life [37]. Another reason to limit the battery downsizing is to make sure that there is enough reserved storage capacity for the battery as a precaution for unexpected situations, such as a bus passing over a charging station without stopping to pick up passengers.

There is potential to further reduce the energy and GHG emissions of the wireless charging system by improving the utilization rates of off-WCs. The yearly utilization rate (\%) and theoretical life (year) of chargers are summarized in Table 1. A wireless charger is assumed to have the same working hours in the total life as a plug-in charger. Based on experience that a typical plug-in charger could work 8 hours per day for 20 years, 58,400 hours is used as the total working hours for both plug-in and wireless chargers. The techno-economic life of on-WCs, off-WCs and plug-in chargers is assumed to be 24 years, though the theoretical life could be longer. Beyond this time frame, there assumes to be technical innovation and better chargers could retire the old ones. The yearly utilization rates of off-WCs located at key bus stops in downtown and suburb are $2 \%$ and $1 \%$, respectively, which are much lower than the off-WCs located at other places like transit centers. In the model, the off-WCs are exclusively used by TheRide bus system. However, the off-WCs can be shared by other fleets, such as the school buses at the University of Michigan commuting between the Central Campus near Ann Arbor downtown and the North Campus in the suburban area. If the charger life is still 24 years, some of the energy and environmental burdens of off-WCs can be allocated to other fleet 
systems. Moreover, due to the unpredictable market prices of wireless chargers during the research stage, this work does not consider cost factors. Future work may take into account these economic metrics and investigate the optimal deployment of off-WCs.

Additionally, a further sensitivity analysis on the charger techno-economic life shows that if both plug-in and wireless chargers are retired after about 17 years of service, there is no difference found in the total GWI between the two systems.

Table 1. Charger yearly utilization and theoretical life.

\begin{tabular}{|c|c|c|c|c|c|c|c|}
\hline \multirow[b]{2}{*}{ Charger category } & \multirow{2}{*}{$\begin{array}{c}\text { On-board } \\
\text { Wireless } \\
\text { Charger }\end{array}$} & \multicolumn{5}{|c|}{ Off-board Wireless Charger } & \multirow{2}{*}{$\begin{array}{l}\text { Plug-in } \\
\text { Charger }\end{array}$} \\
\hline & & Downtown & Suburb & $\begin{array}{c}\text { Parking } \\
\text { lot }\end{array}$ & $B T C$ & $Y T C$ & \\
\hline Operating hours in a year & 1789 & 182 & 96 & 726 & 2065 & 1589 & 1796 \\
\hline Yearly utilization rate & $20 \%$ & $2 \%$ & $1 \%$ & $8 \%$ & $24 \%$ & $18 \%$ & $21 \%$ \\
\hline Theoretical life (years) & 33 & 320 & 606 & 85 & 28 & 37 & 33 \\
\hline
\end{tabular}

Notes: BTC $=$ Blake Transit Center in Ann Arbor; YTC $=$ Ypsilanti Transit Center; Yearly utilization rate $=$ operating hours in a year/8760 hours; Theoretical life (years) $=$ life of a charger (hours)/operating hours in a year.

Future studies could include the bus shell and subsystems in the model boundary to further examine the weight reduction. Secondary vehicle weight reduction, or the vehicle weight reduction due to subsystem resizing, including powertrain, can provide further lightweighting benefits [33, 42-44]. However, the compounding effect of secondary weight reduction is not included due to lack of data on the subsystem of an electric bus. Further studies could also investigate the alternative battery chemistries, such as lithium iron phosphate (LFP), instead of $\mathrm{LiMn}_{2} \mathrm{O}_{4}$ (LMO) in our model. LFP has a long cycle life expectancy of 6,000 cycles at $80 \%$ depth of discharge [5], which may extend the battery life. If available, it can be beneficial to use a U.S.-specific life cycle inventory of LFP. Moreover, the effect of frequent SOC fluctuations on wireless battery life needs to be investigated. A battery/ultracapacitor hybrid energy storage system is recommended for future studies to isolate the battery from frequent charges and extend battery life [45]. Furthermore, the wireless and plug-in battery electricity depleting rates, $k_{1}$ and $k_{2}$ $(\mathrm{kWh} / \mathrm{km})$, are assumed to be constant. However, in reality there is variation in energy consumption in different seasons. Additional energy consumption to maintain comfortable cabin temperatures in the winter and summer would increase the battery electricity depletion rates and this would require a bigger battery to maintain the increased daily energy demand. Future studies can model the actual battery electricity depletion if the battery discharge data for buses in different seasons are available.

Finally, the wireless charging efficiency and grid carbon intensity are key parameters that determine the life cycle energy consumption and GHG emissions. Improving the wireless charging efficiency and "greening" the grid during daytime are key strategies to enhance the energy and environmental benefits of the wireless charging system. 


\section{Acknowledgements}

The authors would like to thank Professor Chris Mi's lab members Tianze Kan, Junjun Deng, Weihan Li and Fei Lu at the University of Michigan-Dearborn for help on charger samples and valuable comments; Peter Hoffman at Delphi Automotive for providing the 2013 Chevrolet Volt charger and valuable comments; Jeff White at Denso for valuable comments; Julia Roberts at Ann Arbor Area Transportation Authority for valuable advice on bus system parameters; and Helaine Hunscher, Hua Cai and Arman Golrokhian in Center for Sustainable Systems at the University of Michigan for valuable comments. This research is part of the U.S.-China Clean Energy Research Center (CERC) on Clean Vehicles, which is partially supported by the U.S. Department of Energy (Award No. DE-PI0000012) and its industrial partners. This research was conducted as part of the Energy Systems Analysis, Technology Roadmaps and Policy thrust of the CERC.

\section{Glossary}

CED: Cumulative Energy Demand

DTD $(\mathrm{km})$ : daily travel distance

ECR: energy consumption rate $(\mathrm{kWh} / \mathrm{km})$

EV: electric vehicle

GHGs: Greenhouse Gases

GWI: Global Warming Impact

ICEV: internal combustion engine vehicle

LCA: Life Cycle Assessment

LFP: lithium iron phosphate

LMO: $\mathrm{LiMn}_{2} \mathrm{O}_{4}$

NOR: No Operation Region

off-WC: off-board portion of the wireless charger (installed on the ground)

on-WC: on-board portion of the wireless charger

OR: Operating Region

OSM: Overcharge Safety Margin

PC: plug-in charger

RSC: Reserved Storage Capacity

SOC: state of charge $(\%)$

SOCR $(\%)$ : the percentage of the $O R(\mathrm{kWh})$ relative to the whole capacity of a new battery $(\mathrm{kWh})$

WPT: Wireless Power Transfer

\section{References}


[1] U.S. EPA. Inventory of U.S. greenhouse gas emissions and sinks: 1990-2011. Washington, DC, USA: U.S. Environmental Protection Agency; 2013.

[2] Davis SC, Diegel SW, Boundy RG. Transportation energy data book. 32nd ed. U.S. Department of Energy (DOE), Oak Ridge National Lab; 2013.

[3] Hawkins TR, Gausen OM, Strømman AH. Environmental impacts of hybrid and electric vehicles—a review. Int J Life Cycle Assess 2012;17(8):997-1014.

[4] BYD Auto Company. 2013 BYD 40-ft electric bus specs $<$ www.byd.com/na/auto/ElectricBus.html>; 2013 [Accessed May 2014].

[5] Majeau-Bettez G, Hawkins TR, Strømman AH. Life cycle environmental assessment of lithium-ion and nickel metal hydride batteries for plug-in hybrid and battery electric vehicles. Environ Sci Technol 2011;45(10):4548-54.

[6] Reikes J at BYD Motors, Inc. Personal communication; July 21, 2014.

[7] Tesla N. Art of transmitting electrical energy through the natural mediums. U.S. Patent 787412; April 18, 1905.

[8] Tesla N. Apparatus for transmitting electrical energy. U.S. Patent 1119732; December 1, 1914.

[9] Suh IS, Kim J. Electric vehicle on-road dynamic charging system with wireless power transfer technology. In: 2013 IEEE international electric machines \& drives conference (IEMDC). IEEE; 2013, p. 234-40.

[10] Nguyen TD, Li S, Li W, Mi CC. Feasibility study on bipolar pads for efficient wireless power chargers. In: Applied Power Electronics Conference and Exposition (APEC), 2014 Twenty-Ninth Annual IEEE. IEEE; 2014, p. 1676-82.

[11] Wu HH, Gilchrist A, Sealy K, Israelsen P, Muhs J. A review on inductive charging for electric vehicles. In: 2011 IEEE international electric machines \& drives conference (IEMDC). IEEE; 2011, p. 143-7.

[12] Onar OC, Miller JM, Campbell SL, Coomer C, White C, Seiber LE. Oak Ridge National Laboratory wireless power transfer development for sustainable campus initiative. In: 2013 IEEE transportation electrification conference and expo (ITEC). IEEE; 2013, p. 1-8.

[13] Wu HH, Gilchrist A, Sealy KD, Bronson D. A high efficiency $5 \mathrm{~kW}$ inductive charger for EVs using dual side control. IEEE Trans Ind Informat 2012;8(3):585-95.

[14] Suh IS, Gu Y. Application of Shaped Magnetic Field in Resonance (SMFIR) technology to future urban transportation. In: CIRP design conference 2011. 2011, p. 226-32.

[15] ISO. ISO 14040 environmental management - life cycle assessment - principles and framework; 2006.

[16] Ning P, Miller JM, Onar OC, White CP. A compact wireless charging system for electric vehicles. In: 2013 IEEE energy conversion congress and exposition (ECCE). IEEE; 2013, p. 3629-34.

[17] Lee WY, Huh J, Choi SY, Thai XV, Kim JH, Al-Ammar EA, et al. Finite-width magnetic mirror models of mono and dual coils for wireless electric vehicles. IEEE Trans Power Electron 2013;28(3):1413-28.

[18] Li S, Mi C. Wireless power transfer for electric vehicle applications. IEEE J Emerg Sel Topics Power Electron 2014;PP(99).

[19] Budhia M, Boys JT, Covic GA, Huang CY. Development of a single-sided flux magnetic coupler for electric vehicle IPT charging systems. IEEE Trans Ind Electron 2013;60(1):31828.

[20] Hischier R, Weidema B, Althaus H, Bauer C, Doka G, Dones R, et al. Implementation of life cycle impact assessment methods. final report Ecoinvent v2. 2 No. 3. Dübendorf, Switzerland: Swiss Centre for Life Cycle Inventories; 2010.

[21] PRé Consultants. SimaPro database manual methods library. PRé Consultants; 2014.

[22] IPCC. Climate change 2013: the physical science basis. working group I contribution to the fifth assessment report of the Intergovernmental Panel on Climate Change. The Intergovernmental Panel on Climate Change (IPCC); 2013. 
[23] Ann Arbor Area Transportation Authority. Route schedules and maps (Winter 2014, effective January 26, 2014 - May 3, 2014). Michigan, USA: Ann Arbor Area Transportation Authority; 2014.

[24] Batterman J, Homa B, Hori S, Jongsma K, Li M, Mani G, et al. Let's roll: reimagining transit on Washtenaw Avenue. Michigan, United States: Taubman College of Architecture \& Urban Planning, University of Michigan; 2012.

[25] Clark NN, Zhen F, Wayne WS, Lyons DW. Transit bus life cycle cost and year 2007 emissions estimation. Washington, DC, USA: Federal Transit Administration; 2007.

[26] Cooney G, Hawkins TR, Marriott J. Life cycle assessment of diesel and electric public transportation buses. J Ind Ecology 2013;17(5):689-99.

[27] Goedkoop M, Oele M, Leijting J, Ponsioen T, Meijer E. Introduction to LCA with SimaPro. PRé Consultants; 2013.

[28] Marano V, Onori S, Guezennec Y, Rizzoni G, Madella N. Lithium-ion batteries life estimation for plug-in hybrid electric vehicles. In: IEEE vehicle power and propulsion conference, 2009 VPPC'09 IEEE; 2009, p. 536-43.

[29] Dunn JB, Gaines L, Sullivan J, Wang MQ. Impact of recycling on cradle-to-gate energy consumption and greenhouse gas emissions of automotive lithium-ion batteries. Environ Sci Technol 2012;46(22):12704-10.

[30] U.S. Department of Transportation. 2013 status of the nation's highways, bridges, and transit: Conditions \& performance. U.S. Department of Transportation Federal Highway Administration \& Federal Transit Administration; 2013.

[31] Kim HC at Ford Motor Company. Personal communication; March 25, 2014.

[32] Kim HC, Wallington TJ. Life cycle assessment of vehicle lightweighting: a physics-based model of mass-induced fuel consumption. Environ Sci Technol 2013;47(24):14358-66.

[33] Lewis AM, Kelly JC, Keoleian GA. Vehicle lightweighting vs. electrification: life cycle energy and GHG emissions results for diverse powertrain vehicles. Appl Energy 2014;126:13-20.

[34] Argonne National Laboratory. Autonomie. UChicago Argonne, LLC; 2009.

[35] Dunn JB, Gaines L, Barnes M, Sullivan J, Wang M. Material and energy flows in the materials production, assembly, and end-of-life stages of the automotive lithium-ion battery life cycle. Argonne National Laboratory; 2012.

[36] Notter DA, Gauch M, Widmer R, Wager P, Stamp A, Zah R, et al. Contribution of Li-ion batteries to the environmental impact of electric vehicles. Environ Sci Technol 2010;44(17):6550-6.

[37] Xu B, Andersson G, Oudalov A, Ulbig A. Degradation-limiting optimization of battery energy storage systems operation: ETH Zurich; 2013.

[38] National Renewable Energy Laboratory. U.S. LCI database project - user's guide. Golden, CO, USA: NREL; 2004.

[39] Zackrisson M, Avellán L, Orlenius J. Life cycle assessment of lithium-ion batteries for plugin hybrid electric vehicles-critical issues. J Clean Prod 2010;18(15):1519-29.

[40] Doucette RT, McCulloch MD. Modeling the $\mathrm{CO}_{2}$ emissions from battery electric vehicles given the power generation mixes of different countries. Energy Policy 2011;39:803-11.

[41] Thornton J. Pulling power from the road: Charged by the route it follows, an electric bus gets a real world test. Mech Eng: ASME; 2014. p. 44-9.

[42] Lewis AM. The potential of lightweight materials and advanced engines to reduce life cycle energy and greenhouse gas emissions for ICVs and EVs using design harmonization techniques: University of Michigan; 2013.

[43] Shiau CSN, Samaras C, Hauffe R, Michalek JJ. Impact of battery weight and charging patterns on the economic and environmental benefits of plug-in hybrid vehicles. Energy Policy 2009;37(7):2653-63. 
[44] Wohlecker R, Johannaber M, Espig M. Determination of weight elasticity of fuel economy for ICE, hybrid and fuel cell vehicles. SAE Technical Paper 2007-01-0343 2007.

[45] Cao J, Emadi A. A new battery/ultra-capacitor hybrid energy storage system for electric, hybrid and plug-in hybrid electric vehicles. In: IEEE vehicle power and propulsion conference, 2009 VPPC '09. IEEE; 2009, p. 941-6. 


\section{Table Captions}

Table 1. Charger yearly utilization and theoretical life.

\section{Figure Captions}

Figure 1. Comparison of wireless charger (a) and plug-in charger (b) for electric vehicles. Component difference is marked in grey. On-board portion of each charger is in the dashed box, and the rest is outside the vehicle. This graph is based on a $6 \mathrm{~kW}$ wireless charger from Professor Chris Mi's laboratory in University of Michigan, Dearborn.

Figure 2. Battery downsizing calculation. Start from a plug-in charged battery to quantify the minimum electricity needed at start of day for a wirelessly charged battery. (1) Operating Region (kWh) of plug-in charged battery; (2) Primary capacity reduction (kWh) due to wireless charging availability; (3) Secondary capacity reduction (kWh) due to fuel economy improvement; (4) Minimum electricity needed (kWh) at start of day for a wirelessly charged battery (not yet including its own Overcharge Safety Margin, Reserved Storage Capacity and No Operation Region); (5) Daily energy saving (batteryto-wheel) due to wireless charging (kWh).

Figure 3. Life cycle energy transmission and conversion cascade.

Figure 4. Cumulative Energy Demand (a) and 100-year Global Warming Impact (b) of plug-in and wireless charging electric bus systems.

Figure 5. Battery weights and energy consumption rates for plug-in and wirelessly charged buses (the columns are in primary y-axis on the left and the line is in secondary $\mathrm{y}$-axis on the right). Blue routes = Ann Arbor city routes; red routes = Ann ArborYpsilanti intercity routes; green routes $=$ Ypsilanti city routes.

Figure 6. Sensitivity analysis of key parameters. Parameters are ranked according to sensitivity. $(\mathrm{PC}=$ greenhouse gas emissions from plug-in charging system; $\mathrm{WC}=$ greenhouse gas emissions from wireless charging system) 


\section{Graphical Abstract:}

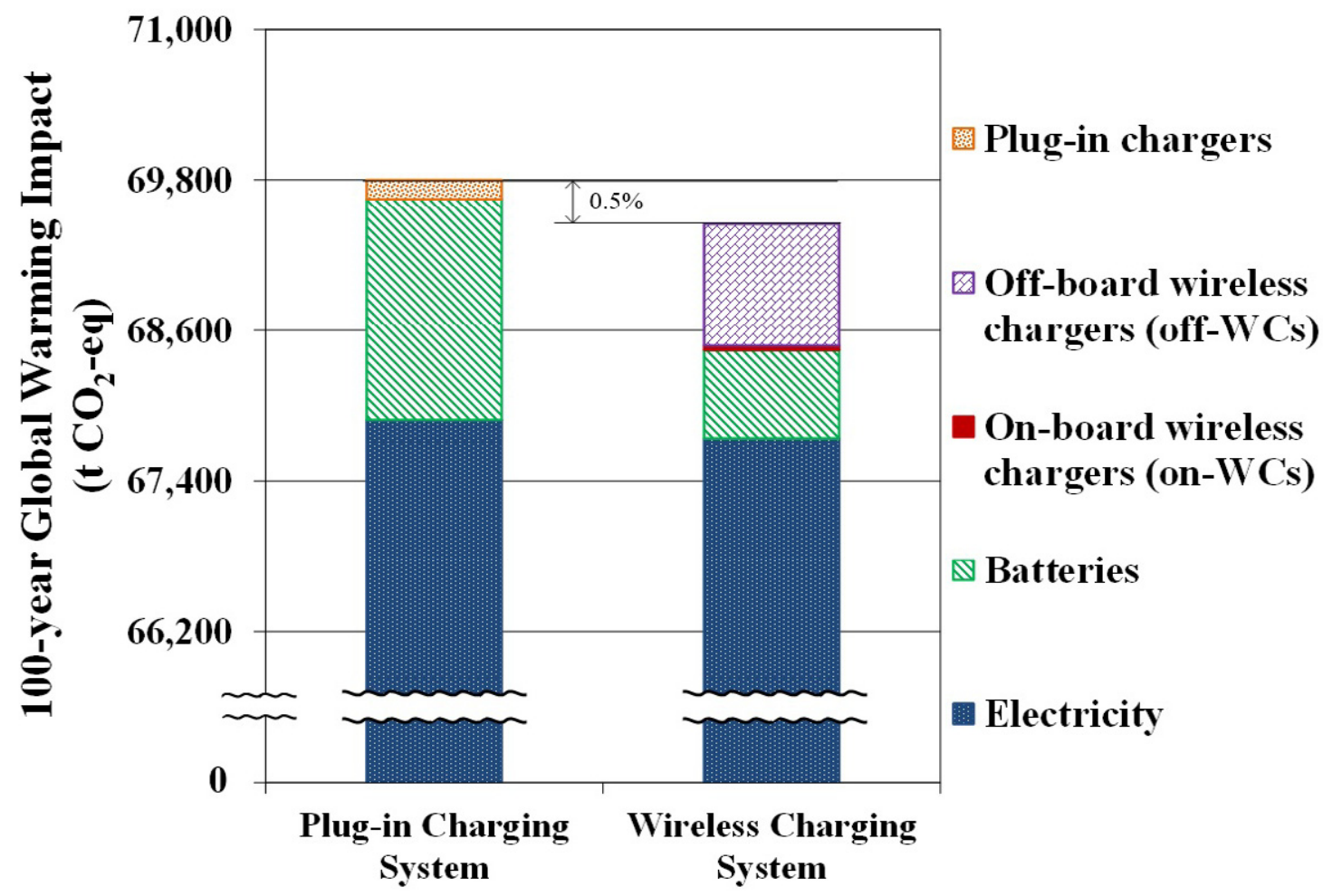

In this study, plug-in and wireless charging for an all-electric bus system are compared from the life cycle energy and greenhouse gas (GHG) emissions perspectives. The comparison of life cycle GHG emissions is shown in the graph above. The major differences between the two systems, including the charger, battery and use-phase electricity consumption, are modeled separately and compared aggregately. In the base case, the wireless charging system consumes $0.3 \%$ less energy and emits $0.5 \%$ less greenhouse gases than plug-in charging system in the total life cycle. To further improve the energy and environmental performance of the wireless charging system, key parameters including grid carbon intensity and wireless charging efficiency are analyzed and discussed in this paper. 